\title{
Neue Herzchirurgie am Freiburger Spital?
}

\section{Michele Genoni ${ }^{a}$, Mario Stalder ${ }^{b}$}

a Prof. Dr. med., Präsident Schweizerische Gesellschaft für Herz- und thorakale Gefässchirurgie (SGHC)

b PD Dr. med., Sekretär SGHC

\author{
Mittel- und langfristig gefährdet die Eröffnung neuer herzchirurgischer Abteilun- \\ gen in der Schweiz die medizinische Versorgung im Allgemeinen und führt zu \\ beträchtlichen Einbussen in der Qualität der Medizin.
}

«Mit Herzoperationen verdienen die Schweizer Spitäler gutes Geld. Auch das Spital Fribourg HFR will nun in den lukrativen Markt einsteigen und eine eigene herzchirurgische Klinik eröffnen", schreibt die Sonntagszeitung. Und die Schweiz am Sonntag berichtet: «Im Kanton Fribourg will das Kantonsspital HFR eine eigene Herzchirurgie aufbauen und ist dafür - sehr zum Ärger des Inselspital Berns - eine Kooperation mit dem CHUV in Lausanne eingegangen.» In der Tat ist die Herzchirurgie am Freiburger Spital in voller Planung. Dabei müssen medizinische, ökonomische und politische Aspekte berücksichtigt werden. Freiburg selbst ist für einen Alleingang zu klein. Das Spital möchte keine komplexen Fälle behandeln, sondern in erster Linie die Universitätsspitäler entlasten.

\section{Besteht ein Überangebot?}

In der Schweiz wird bisher an 18 Zentren eine herzchirurgische Abteilung/Klinik betrieben. Bezüglich Qualität müssen diese herzchirurgischen Zentren einen Vergleich mit Kliniken in Europa nicht scheuen. Dennoch muss sich die Fachgesellschaft der Diskussion stellen, ob 18 herzchirurgische Zentren für die kleine Schweiz nicht zu viel sind oder ob nicht bereits heute ein Überangebot besteht.

\section{Das Wohl der Patienten steht im Vordergrund.}

Sicherlich kann der Föderalismus als eines der Standbeine der erfolgreichen Schweizer Geschichte betrachtet werden. Dennoch ist zu fragen, ob eine gewisse Regulation nicht nötig ist, um die Qualität zu garantieren beziehungsweise die Gesundheitskosten in den Griff zu bekommen. Dabei steht das Wohl der Patienten im Vordergrund: Sie haben nach hochkomplexen Eingriffen bessere Chancen, wenn der Chirurg und sein Team erfahren sind und regelmässig Operationen durchführen. Wie viele Eingriffe ein Chirurg jährlich vornimmt, ist mitunter ein entscheidendes Qualitätskriterium. Das Bundesamt für Gesundheit (BAG) drängt im Rahmen von «Gesundheit 2020» darauf, hochkomplexe Eingriffe auf wenige Zentren zu konzentrieren. Die Kantone sind an sich gesetzlich verpflichtet, nicht nach kantonalen Wirtschaftsinteressen zu planen, sondern gesamtschweizerisch zu denken.

\section{Fachgesellschaft mit Qualitätsstrategie}

Die Schweizerische Gesellschaft für Herz- und thorakale Gefässchirurgie (SGHC) mischt sich in Konzentrationsprozesse nicht direkt ein. Vielmehr hat sie versucht, mit einer Qualitätsstrategie eine Diskussionsbasis zu diesem Thema zu schaffen. Dies mit der Überlegung, dass jede Klinik weiterbestehen kann, wenn die Qualitätsvorgaben der Fachgesellschaft erfüllt werden.

\section{Wie viele Eingriffe ein Chirurg jährlich} vornimmt, ist mitunter ein entscheidendes Qualitätskriterium.

Die Ausweitung des herzchirurgischen Angebots in der Schweiz gefährdet jedoch die Einhaltung europäischer Richtlinien der Fachgesellschaften EACTS und ESC: Kämpft nun mit Freiburg eine weitere Klinik um Patienten, sinken die Fallzahlen in anderen Spitälern. Bereits heute kann nur eine Minderheit der herzchirurgischen Kliniken die empfohlenen Mindestfallzahlen von 200 Bypassoperationen pro Klinik und Jahr erfüllen. Zu bedenken gilt es jedoch, dass die Qualität nur bedingt von den Fallzahlen abhängt. Die Qualitätsstrategie berücksichtigt auch diese besonderen schweizerischen Gegebenheiten. 


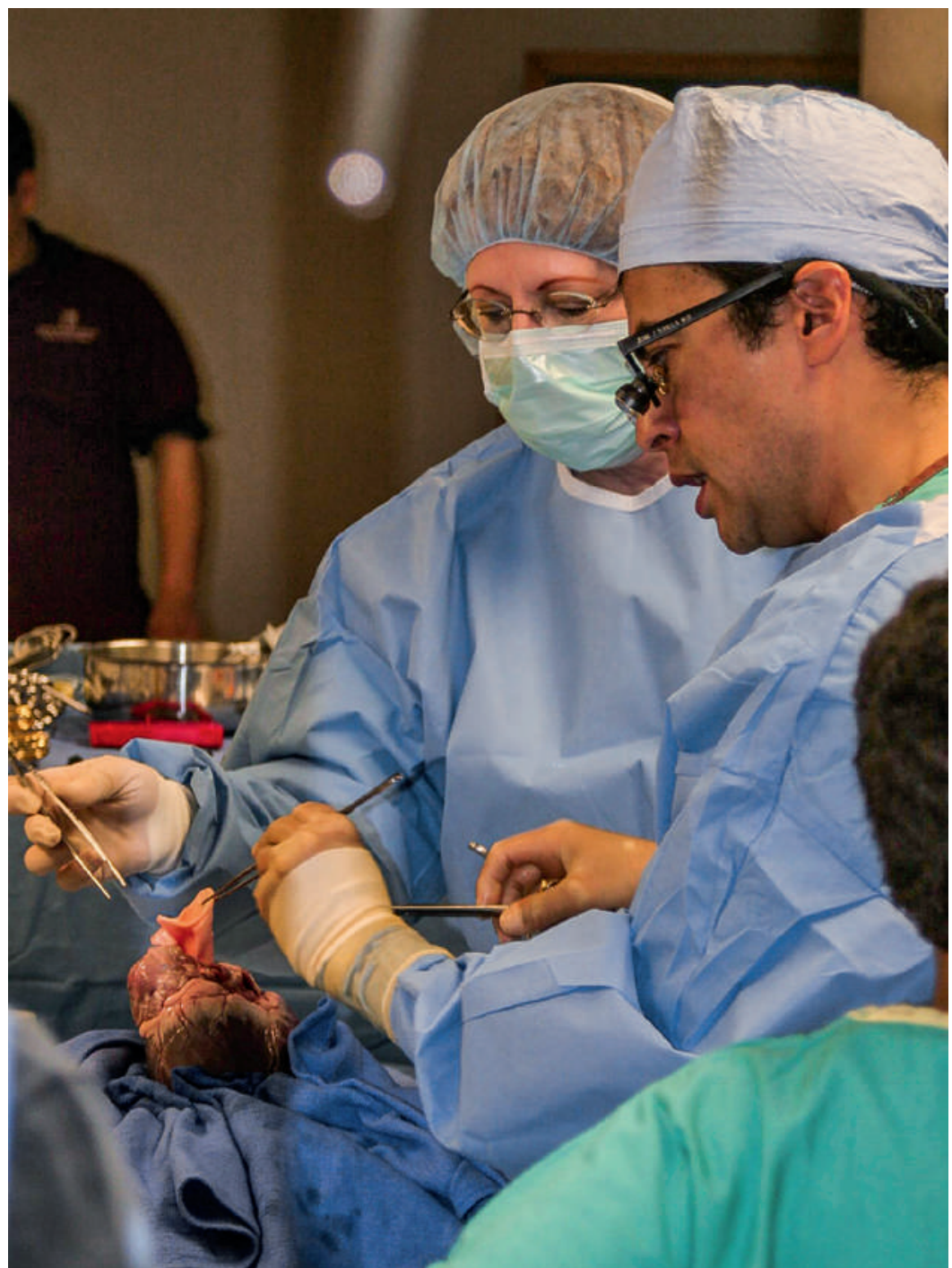

Bereits heute kann nur eine Minderheit der herzchirurgischen Kliniken der Schweiz die von der Europäischen Fachgesellschaft empfohlenen Mindestfallzahlen von 200 Bypassoperationen pro Klinik und Jahr erfüllen.

\section{Zentren mit hohen Eingriffszahlen notwendig}

Korrespondenz: Prof. Dr. med. Michele Genoni Stadtspital Triemli Birmensdorferstrasse 497 CH-8063 Zürich
Ein wichtiger Aspekt betrifft den Blick in die Zukunft: Der Mangel an herzchirurgischem Nachwuchs ist in der Schweiz und Westeuropa eminent. Die SGHC hat sich zum Ziel gesetzt, die Ausbildung junger Kollegen zu fördern, Ausbildungscurricula zu erarbeiten und die Umsetzung in den Ausbildungskliniken zu unterstützen. In Kooperation mit der deutschen Gesellschaft werden sogenannte Fach-Module für Nachwuchskräfte angeboten. Für eine qualitativ hochstehende Ausbildung von Herzchirurgen sind allerdings Zentren mit einer hohen Zahl an Eingriffen, einem weiten Spektrum der Herzchirurgie und fachlich einwandfreien Ausbildern notwendig.

Für eine qualitativ hochstehende Ausbildung von Herzchirurgen braucht es Zentren mit einer hohen Zahl an Eingriffen.

Die Eröffnung eines herzchirurgischen Zentrums wie in Freiburg kann für einen Kanton kurzfristig sehr interessant sein. Es erlaubt dem Spital, das Angebot einer kardiologischen Abteilung zu erweitern. Es ist jedoch zu berücksichtigen, dass zu einer herzchirurgischen Abteilung neben den Herzchirurgen auch spezialisierte Anästhesisten, Intensivmediziner und Pflegefachpersonen gehören. Nur eine eingespielte Zusammenarbeit dieser Disziplinen führt zu hoher Behandlungsqualität. Diese wird erfahrungsgemäss erst nach Jahren erreicht.

Zudem muss ein grosser Maschinenpark angeschafft werden. Mittel- und langfristig gefährdet die Eröffnung neuer herzchirurgischer Abteilungen die medizinische Versorgung im Allgemeinen und führt zu beträchtlichen Einbussen in der Qualität der Medizin. Denn die Ressourcen jeglicher Art werden immer knapper. Diese Argumente müssen in die Erwägungen zur geplanten Eröffnung einer herzchirurgischen Abteilung im Spital Freiburg berücksichtigt werden.

Letztlich geht es darum, die notwendigen Qualitätsstandards einhalten zu können. Die SGHC verfügt diesbezüglich über ein ausgeprägtes Know-how, das sie gerne in die Diskussion über herzchirurgische Qualitätsfragen einbringt.

\section{Bildnachweis}

(c) akeg | Eric Schmuttenmaer from Wheaton, USA | wikimedia.org 